\title{
Comparison of the Effect of Cooling the Lower Abdomen and Oxytocin on Postpartum Blood Loss in Vaginal Delivery
}

\author{
Zeinab Zarabadipour $^{1}{ }^{\circledR}$, Hamideh Pakniat $^{2}$, Masoumeh Rezaei Niaraki ${ }^{1}$, Nezal Azh $^{3 *}$
}

\begin{abstract}
Objectives: Using uterotonic drugs to prevent postpartum hemorrhage is recommended in the health centers. However, the related studies are contradictory, thus the evaluation of non-invasive methods with minimal side-effects such as icepack would be useful. Traditionally, icepack has been applied, along with drugs in most deliveries in postpartum hemorrhage (PPH) in Iran although it has not evidenced yet. Therefore, the present study aimed to evaluate the effect of the icepack on blood loss.

Materials and Methods: This study was a randomized controlled trial. The sample size included 58 women including 29 intervention and 29 control groups. The intervention group benefited from an icepack placed on the lower abdomen 2 hours after placental delivery while the control group received 20 units of oxytocin. Subsequently, the amount of lost blood after placental delivery was measured via weighting the sheets and pads. The mean difference between the two groups was detected with $80 \%$ power at the two tails of a $5 \%$ significance level.

Results: Based on the results, there was no significant difference between the groups in parity, neonatal weight, and hemoglobin levels before and after delivery in the second and third stage of delivery and breastfeeding. Moreover, excessive PPH was $12.1 \%(17.2 \%$ vs. $6.9 \%)$. Thus, no significant difference $(P>0.05)$ was observed between the groups in terms of blood loss $(254 \pm 68 \mathrm{~mL}$ vs. $245 \pm$ $53 \mathrm{~mL}$, respectively) although the only complication in the intervention group was an unpleasant cold feeling in the abdominal area. Conclusions: The application of the icepack in low-risk women is a non-pharmacological and affordable method that can be a good alternative to oxytocin in order to decrease blood loss after delivery.

Keywords: Icepack, Lower abdomen, Blood loss, PPH
\end{abstract}

\section{Introduction}

Postpartum hemorrhage (PPH) refers to the blood loss of more than $500 \mathrm{cc}$ after completing the third stage of labor $(1,2)$. Nonetheless, this estimation is generally only half of the actual blood loss based on the evidence (3). In addition, PPH is known as one of the major reasons for maternal mortality (4). In other words, $28 \%$ and $10 \%$ of maternal deaths in developing and developed countries are related to $\mathrm{PPH}$, respectively $(2,5,6)$. Further, $\mathrm{PPH}$ is characterized by a $10 \%$ drop in hematocrit levels and the need for postpartum blood transfusions (7). It could lead to hypovolemic shock, disseminated intravascular coagulation; and acute renal failure $(8,9)$.

Treatments should address the causes of PPH. The possible causes of uterine postpartum bleeding are trauma, retained placenta, and uterine atony (10-12). Uterotonic drugs such as oxytocin, ergo derivatives, and misoprostol are used as the first line of treatments for $\mathrm{PPH}$ in Iran (7). Except for uterine massage, the other treatments of PPH have disadvantages on the mother (e.g., headache, vomiting, the elevation of blood pressure, and the like) or breastfeeding $(1,13)$. Consequently, preventing $\mathrm{PPH}$ is highly important. Reducing hemorrhage is usually implemented by the routine active management of placenta delivery by drug using to contract the uterus such as oxytocin (11). Today, ice therapy is used as one of the new methods for the treatment of many diseases, even cancer by lessening pain, inflammation, and vasoconstriction (14). It can be one of the useful methods for preventing uterine atony and PPH (15).

Based on the evidence, placing an icebag on the lower abdomen for cooling the uterus is one of the standard non-pharmacological prophylactic strategies to prevent PPH in low-risk women in Japan (15), and its argument is that cold compresses would contract the myometrium and decreases the amount of blood flow (16). In contrast, some studies have reported conflicting results about the effectiveness of icebag in preventing uterine atony in normal delivery $(16,17)$.

This study was designed to reduce maternal deaths as an important factor for children's growth, specifically in poor countries (18) by using available methods such 
Key Messages

- Blood loss is a life threatening complication after birth so emergency interventions are need.

- Although icebag is a traditionally available intervention to reduce blood loss, there is little evidence to support its effectiveness.

as the icebag. Meanwhile, in Iran, prophylactic oxytocin (10-20 units) is administrated during the third stage of labor (19), and icebag along with other uterotonic agents is used in the treatment of PPH while there is no evidence for separately detecting the effectiveness of this method. Moreover, the conflicting results in some studies on the effect of the routine use of synthetic drugs (1) and noninvasive therapeutic methods such as cooling lower abdominal need further assessment.

\section{Materials and Methods}

The study as a parallel, double-blind clinical trial determined the effect of the ice bag on the amount of blood loss and prevention of $\mathrm{PPH}$ in the first two hours after delivery. The design of the project strictly complied with the Guidelines for Reporting Parallel Group Randomized Trials (20).

This study was conducted in the labor wards of 3 hospitals in Qazvin, Iran, from February 20, 2019 to November 26, 2019. The subjects were women with normal vaginal delivery, with immediate placenta removal up to 2 hours. The study criteria were maternal aged 18-35 years, term pregnancy, alive and singleton pregnancy, fetal weight in the range of 2500-4000 g, gestational age between 37 and 42 weeks, vertex presentation, and normal second and third stage length. On the other hand, the exclusion criteria included pre-eclampsia, polyhydramnios, body mass index (BMI) more than 40, the disorder of blood coagulation, use of any anticoagulants, hepatic dysfunction, placental abruption, cesarean section birth, history of prenatal hemorrhage, rapid delivery less than 3 hours, deep and large laceration (grade 3 or 4), use of oxytocin at any stage of labor, previous cesarean section, or uterine surgery.

\section{Randomization and Blinding}

The sample size was 58. The intervention (cooling of the lower abdomen) and control (oxytocin) groups were randomly assigned during placenta delivery using block 4 th allocation. Then, 13 blocks were made, letters A and B were written for the intervention and control groups (e.g., $\mathrm{AABB}, \mathrm{ABAB}$, and the like), respectively, and then were put into a bag. They were picked up one by one by a ward clerk, and finally, the sequences of the blocks were written and assigned to the intervention and control groups, respectively.

The participants were masked by a light sandbag the same as an ice bag. Therefore, the participant and data analyzers did not know what type of intervention they received although caregivers could not be masked due to the nature of this intervention.

\section{Procedure}

Before the study, midwives received training about who, when, and where to place the icebag to cool the abdomen, then after placental removal, uterine massage, and clot removal, the control group received 20 units of oxytocin in $1 \mathrm{~L}$ of Ringer serum according to the health ministry protocol (19), and a light sandbag weighing $385 \mathrm{~g}$ (9.16Ib) was placed on the uterus above pubis symphysis.

In the intervention group, the icebag was placed on the lower abdomen. Each icepack was frozen under $-18^{\circ} \mathrm{C}$ temperature for more than 8 hours. In addition, women in both groups were similarly managed by midwives and obstetricians. If the participants had abnormal bleeding, the midwives and obstetricians treated them based on determined protocols provided by the individual physician in both groups.

\section{Outcome}

The first and second outcomes were the rate of $\mathrm{PPH}$ and the amount of blood loss, respectively. $\mathrm{PPH}$ was determined as need transfusion or other management according to obstetrician diagnosis (19).

\section{Data Collection}

A checklist was used to record the data including delivery type, duration of the second and third stages, complications such as excessive $\mathrm{PPH}$, hemoglobin, neonatal weight, neonatal gender, blood pressure, temperature, and pulse. The respiration rate and uterine contraction were monitored every 15 minutes at the first 2 hours (after placental removal). The duration of lactation and the duration of sucking by the infant were also recorded in this form.

The validity of the checklist was done by the content validity method with 10 midwives and obstetricians' teachers. Interrater methods were used and the coefficient of agreement (0.7) was calculated to obtain the reliability. The Japanese digital scale and weighting scale and the sensitivity of $1 \mathrm{~g}$ were daily checked for half a kilo weight to determine its scientific reliability.

To measure the amount of bleeding, after ensuring complete placental removal and the absence of PPH (as mentioned above), a new mat (Mark Bella, $85 \mathrm{~g} \pm 7 \mathrm{~g}$ absorbent powder and $60 \times 160$ weighted dimensions) was placed under the mother, and then they were given a pad (with a large Mark My Lady weighting $10 \mathrm{~g}$ of 30 $\times 7$ dimensions) for both groups. At the end of the first and second hour after delivery, all pads and mats were weighted by a person unaware of the study in both groups. It should be noted that all gauzes were weighted and recorded in the case of vaginal tampon in perineal 
laceration or episiotomy. Hemoglobin and hematocrit were routinely checked at admission and three hours after birth.

Data Analysis

Data were analyzed by the SPSS software (version 24, IBM, Armonk New York, USA). The $t$ test was used for the comparison of continuous variables such as the amount of blood loss among the groups. Finally, the Chi-square test was applied to compare dichotomous variables such as the rate of $\mathrm{PPH}$.

\section{Results}

In this study, 58 postpartum women were included and were randomly assigned to oxytocin $(n=29)$ and icebag $(\mathrm{n}=29)$ groups in the third stage of delivery. Two groups were compared in parity, abortion, education, and job. Most of them were literate and housewives and 70\% of women were multipara (Table 1).

The type of delivery and the percentage of episiotomy (41.1\% in icepack versus $51.7 \%$ in the oxytocin group) were similar in both groups. There was no side effect in the oxytocin group except for the pain in 2 women although $52 \%$ of cases of the icebag group had a bad sensation of cold (Table 1). There were no significant differences between the two groups in terms of pre-pregnancy BMI, weight gain during pregnancy, neonatal weight, hemoglobin levels before and after delivery, lactation, and the second and third stage duration. Although the second stage showed significant differences between the primipara and multipara (it is longer in primipara than multipara), they were similar among the groups (Table 2).
The $\mathrm{PPH}$ rate was $12.1 \%(17.2 \%$ and $6.9 \%$ in the intervention and control groups, respectively), and no significant differences were observed between the two groups. Vaginal blood loss was $254 \pm 68.11$ and $245 \pm$ 53.23 in the intervention and control groups, respectively, representing no significant differences between the two groups (Table 3 ).

\section{Discussion}

This study was done on 58 low-risk pregnant women. All samples were matched for the duration of lactation, neonatal weight, and socioeconomic factors affecting $\mathrm{PPH}$, and the findings revealed no significant difference. Based on the results, the administration of 20 units of oxytocin or the use of an icebag on the lower abdomen (uterine) did not significantly change the rate of uterine bleeding after delivery. Normal blood loss in low-risk women is estimated to be a maximum of $500 \mathrm{~mL}$ (1). The data from this study estimated the mean total blood loss for multiparous women 275 (140-432) and primipara $270(170-590) \mathrm{mL}$ while the mean total blood loss demonstrated no reduction in the intervention group in comparison with the control group (254 vs. 245).

In the Masuzawa et al study in Japan, bleeding was estimated to be 473 in the untreated control and $513 \mathrm{~mL}$ in the intervention group (15), which is much higher than that of the current study. Due to collecting the lost blood method in the third stage after placental removal and uterine massage in the current study (because of ensuring abnormal bleeding), the new mat was placed for collecting blood loss, thus the amount of intrauterine bleeding associated with primary clotting was not measured after

Table 1. Demographic Characteristics of the Participants

\begin{tabular}{|c|c|c|c|c|}
\hline \multirow{3}{*}{ Variable } & & \multicolumn{2}{|c|}{ Groups } & \multirow{3}{*}{$P$ Value } \\
\hline & & \multirow{2}{*}{$\begin{array}{c}\text { Ice Packed } \\
\text { No. }(\%), n=29\end{array}$} & \multirow{2}{*}{$\begin{array}{c}\text { Oxytocin } \\
\text { No. }(\%), n=29\end{array}$} & \\
\hline & & & & \\
\hline Age (year), mean & & $28.17 \pm 7.81$ & $31.20 \pm 4.63$ & 0.08 \\
\hline Maternal body $\mathrm{m}$ & f pregnancy $(\mathrm{kg})$, mean $\pm \mathrm{SD}$ & $25.40 \pm 4.20$ & $24.75 \pm 2.87$ & 0.51 \\
\hline Maternal weight & mean $\pm \mathrm{SD}$ & $10.45 \pm 3.18$ & $10.90 \pm 4.26$ & 0.67 \\
\hline \multirow{4}{*}{ Parity } & First & $8(27.6)$ & $9(31.0)$ & \multirow{4}{*}{0.67} \\
\hline & Second & $10(34.5)$ & $12(41.4)$ & \\
\hline & Third & $5(17.2)$ & $4(13.8)$ & \\
\hline & Fourth & $6(20.7)$ & $4(13.8)$ & \\
\hline \multirow{2}{*}{ Abortion history } & No & $21(72.4)$ & $19(65.5)$ & \multirow{2}{*}{0.12} \\
\hline & Yes & $8(27.5)$ & $10(31.5)$ & \\
\hline \multirow{6}{*}{ Education } & Illiterate & $4(13.8)$ & $1(3.4)$ & \multirow{6}{*}{0.07} \\
\hline & Primary & $6(20.7)$ & $1(3.4)$ & \\
\hline & Secondary & $9(31.0)$ & $10(34.5)$ & \\
\hline & High school & $5(17.2)$ & $10(34.5)$ & \\
\hline & Bachelor & $0(0)$ & $2(6.9)$ & \\
\hline & MA & & & \\
\hline \multirow{3}{*}{ Job } & Housewife & $24(82.8)$ & $20(69.0)$ & \multirow{3}{*}{0.19} \\
\hline & Employee & $4(13.8)$ & $9(31.0)$ & \\
\hline & Hair stylist & $1(3.4)$ & $0(0.0)$ & \\
\hline
\end{tabular}

Note. SD: Standard deviation

As shown, the demographic data of the two groups were not significantly different. 
Table 2. Birth Data

\begin{tabular}{|c|c|c|c|c|}
\hline \multirow{3}{*}{ Variable } & & \multicolumn{2}{|c|}{ Groups } & \multirow{3}{*}{$P$ Value } \\
\hline & & \multirow{2}{*}{$\begin{array}{c}\text { Ice Packed } \\
\text { No }(\%), n=29\end{array}$} & \multirow{2}{*}{$\begin{array}{c}\text { Oxytocin } \\
\text { No }(\%), n=29\end{array}$} & \\
\hline & & & & \\
\hline \multicolumn{2}{|l|}{ Baby weight (Mean \pm SD) } & $3206 \pm 437$ & $3284 \pm 341$ & 0.47 \\
\hline \multicolumn{2}{|c|}{ Breastfeeding time in the first 2 hours $(\mathrm{min})$, mean $\pm \mathrm{SD}$} & $46.58 \pm 22.30$ & $52.85 \pm 18.78$ & 0.57 \\
\hline \multicolumn{2}{|c|}{ Time of the second stage of labor $(\mathrm{min})$, mean $\pm \mathrm{SD}$} & $20.62 \pm 20.28$ & $19.74 \pm 14.72$ & 0.85 \\
\hline \multicolumn{2}{|c|}{ Time of the second stage of labor in primipara $(\mathrm{min})$, mean $\pm S D$} & $39.16 \pm 26.53$ & $34.37 \pm 16.99$ & 0.85 \\
\hline \multicolumn{2}{|c|}{ Time of the second stage of labor multipara (min), mean \pm SD } & $14.44 \pm 13.70$ & $13.57 \pm 8.09$ & 0.85 \\
\hline \multicolumn{2}{|c|}{ Time of the third stage of labor $(\min )$, mean $\pm S D$} & $7.54 \pm 7.40$ & $6.14 \pm 3.31$ & 0.38 \\
\hline \multicolumn{2}{|c|}{ Hemoglobin at admission (g), mean \pm SD } & $12.73 \pm 0.73$ & $12.80 \pm 0.64$ & 0.73 \\
\hline \multicolumn{2}{|c|}{ Hemoglobin 3 hours after delivery $(\mathrm{g})$, mean $\pm \mathrm{SD}$} & $12.71 \pm 0.70$ & $12.71 \pm 0.60$ & 0.74 \\
\hline \multirow{3}{*}{ Type of delivery } & Normal & $12(41.4)$ & $10(34.5)$ & \multirow{3}{*}{0.75} \\
\hline & Episiotomy & $12(41.4)$ & $15(51.7)$ & \\
\hline & Laceration & $5(17.2)$ & $4(13.8)$ & \\
\hline \multirow{2}{*}{ Placental separation } & Schultz & $25(86.2)$ & $26(89.7)$ & \multirow{2}{*}{0.67} \\
\hline & Duncan & $1(13.8)$ & $3(10.3)$ & \\
\hline \multirow{2}{*}{ Baby gender } & Boy & $18(62.1)$ & $14(48.3)$ & \multirow{2}{*}{0.29} \\
\hline & Girl & $11(37.9)$ & $15(51.7)$ & \\
\hline \multirow{2}{*}{ Side effects } & No & $19(65.5)$ & $27(93.1)$ & \multirow{2}{*}{0.01} \\
\hline & Yes & $10(34.5)$ & $2(6.9)$ & \\
\hline \multirow{3}{*}{ Lactation and skin contact } & Immediately after birth & $18(62.1)$ & $14(48.3)$ & \multirow{3}{*}{0.18} \\
\hline & Up to $30 \mathrm{~min}$ & $4(13.8)$ & $10(34.5)$ & \\
\hline & After $30 \mathrm{~min}$ & $7(24.1)$ & $5(17.2)$ & \\
\hline
\end{tabular}

Note. SD: Standard deviation

Based on the data, there is no significant difference regarding lactation, weight gain during pregnancy, and the duration of labor. The type of delivery and the rate of skin contact did not differ significantly between the two groups although the side effect was more considerable in the icebag group (cold feeling).

Table 3. Average Amount of Blood Loss and the Frequency of PPH

\begin{tabular}{|c|c|c|c|c|}
\hline \multirow{3}{*}{\multicolumn{2}{|c|}{ Variable }} & \multicolumn{2}{|c|}{ Groups } & \multirow{3}{*}{$P$ Value } \\
\hline & & \multirow{2}{*}{$\begin{array}{c}\text { Ice pack } \\
\text { No }(\%), \mathbf{n}=\mathbf{2 9} \\
\end{array}$} & \multirow{2}{*}{$\begin{array}{c}\text { Oxytocin } \\
\text { No }(\%), n=29\end{array}$} & \\
\hline & & & & \\
\hline Third stage bloo & & $254.50 \pm 68.11$ & $245.74 \pm 53.23$ & 0.60 \\
\hline \multirow{3}{*}{$\mathrm{PPH}$ frequency } & No & $24(82.8)$ & $27(93.1)$ & \multirow{2}{*}{0.22} \\
\hline & Yes & $5(17.2)$ & $2(6.9)$ & \\
\hline & & Primipara, $n=17$ & Multipara, $n=41$ & \\
\hline \multicolumn{2}{|c|}{ Third stage blood loss $(\mathrm{mg})$, mean \pm SD } & $239 \pm 48.55$ & $275.56 \pm 83.40$ & \multirow{2}{*}{0.41} \\
\hline \multicolumn{2}{|c|}{ Third stage blood loss (mg), minimum - maximum } & $170-590$ & $140-430$ & \\
\hline
\end{tabular}

Note. SD: Standard deviation; PPH:

As shown, no significant difference was observed in the blood loss amount between the two groups although the maximum and minimum bleeding rates were significantly higher in the primipar compared to multiparous women.

placental removal. Overall, their results also showed no significant differences between the two groups, which is in line with the findings of the current study. However, in another study, cooling the lower abdomen was not useful for uterine activity during the two hours after placental delivery (17). In another study performed to evaluate the effect of uterine cooling on cesarean delivery, this method was found to be significantly effective in reducing post-cesarean bleeding (16), which is not in line with the findings of our study. However, cooling the uterus directly in the cesarean section causes shrinkage and reduces bleeding, and thus is more effective compared to indirect cooling beyond the abdominal skin.

Although there is no study in this area in Iran, the icebag is commonly used in combination with other drugs in PPH. A study by Mooshkbid Haghighi et al on postnatal hemorrhage showed that the administration of oxytocin could reduce the blood loss in the first hour after placenta removal compared with non-pharmacologic drugs such as massage ( 39.5 vs. 43.68$)$ but this difference was not significant. They concluded that although blood loss decreased in the oxytocin group in the first hour after delivery, oxytocin administration was unnecessary 24 hours after birth (21). Dabbaghi Gale et al found that oxytocin administration is the same in reducing $\mathrm{PPH}$ in comparison with oral misoprostol (22). Likewise, Mojahed et al suggested the effectiveness of eating dates in preventing $\mathrm{PPH}$ (23). Another study pointed to the 
efficacy of consuming dill (24) and oxygen inhalation (3). The results of these studies corroborate with those of the present study, emphasizing that oxytocin is not the only safe drug for preventing PPH. Nonetheless, Hofmeyr et al reviewed two randomized controlled trials and agreed on uterine massage effectiveness after birth before or after the delivery of the placenta or both for reducing postpartum blood loss. However, the results of this review were inconclusive. The disadvantages of uterine massage were time-consuming for staff time and women discomfort. They emphasized no change in the recommended practice (11), which conforms with the results of the current study because of using a different intervention. It should be noted that the only complication of the icebag on the lower abdomen was the feeling of coldness which was mentioned in the Japanese study (15), this is the only barrier to the use of the icebag.

\section{Conclusions}

Based on the results, in low-risk deliveries, icebags as a non-pharmacological alternative can be as effective as oxytocin in preventing PPH, thus it is recommended for low-risk women who do not wish to use any medication. Finally, midwives could use the icebag without or/along with oxytocin after every vaginal delivery in order to prevent $\mathrm{PPH}$.

\section{Limitations}

To prevent unpredictable $\mathrm{PPH}$, the icebag method was performed cautiously, thus the investigation took a long time. The minimum sample size was estimated as this is a student project.

\section{Suggestion for Future Studies}

We will suggest doing this study on a large sample size and on multiparous women who are more likely to have uterine atony.

\section{Authors' Contribution}

ZZ designed the study; ZZ and RM participated in data collecting; $\mathrm{PH}$ contributed to the data analysis; AN participated in data analysis, article preparation, article submission and study design.

\section{Conflict of Interests}

Authors declare that they have no conflict of interests.

\section{Ethical Issues}

The study protocol was approved by the Research Committee of the School of Nursing and Midwifery with Code of Ethics IR.QUMS.REC.1397.108. Further, this study was registered in the Iranian Clinical Trial System on July 24, 2019 (identifier: IRCT20190430043433N2). The eligible women were informed about the goal of this study both verbally and nonverbally, and all participants provided written informed consent.

\section{Financial Support}

Vice-chancellor for research of Qazvin University of Medical Sciences supported the study.

\section{Acknowledgments}

This study is the result of a student research project approved by the Student Research Committee of the School of Nursing and Midwifery. We appreciate the Research Assistants and the members of the Student Research Committee, as well as all participants in this research. .

\section{References}

1. James D, Steer PJ, Weiner CP, Gonick B, Robson SC. High Risk Pregnansy. 5th ed. United Kingdom: Cambridge University Press; 2017.

2. Cunningham FG, Leveno $\mathrm{K}$, Bloom $\mathrm{E}$, et al. Williams Obstetrice. 25th ed. USA: McGraw-Hill; 2018.

3. Sohrabi Z, Ziagham S, Akbari M, Bastami A. Effect of oxygen inhalation on postpartum hemorrhage. Iran J Obstet Gynecol Infertil. 2015;18(168):1-6. [Persian].

4. Lu MC, Fridman M, Korst LM, et al. Variations in the incidence of postpartum hemorrhage across hospitals in California. Matern Child Health J. 2005;9(3):297-306. doi:10.1007/ s10995-005-0009-3

5. Drife J. Management of primary postpartum haemorrhage. $\mathrm{Br} J$ Obstet Gynaecol. 1997;104(3):275-277. doi:10.1111/j.1471-0528.1997.tb11453.x

6. Fazel N, Esmaeili H, Shamaeyan Razavi N. Effect of cumin oil on post partum hemorrhage after cesarean. Iranian Journal of Medicinal and Aromatic Plants. 2013;29(1):97-104. [Persian].

7. Fakour F, Mirzayi M, Naghipour MR, Ebrahimi H, Mahdavi M. Comparison between sublingual misoprostol and intravenous oxytocin in management of third stage of labor [Persian]. Iran J Obstet Gynecol Infertil. 2013;15(34):7-14. doi: 10.22038/ ijogi.2015.6118

8. Sheikh L, Najmi N, Khalid U, Saleem T. Evaluation of compliance and outcomes of a management protocol for massive postpartum hemorrhage at a tertiary care hospital in Pakistan. BMC Pregnancy Childbirth. 2011;11:28. doi:10.1186/1471-2393-11-28

9. Hoveyda F, MacKenzie IZ. Secondary postpartum haemorrhage: incidence, morbidity and current management. BJOG. 2001;108(9):927-930. doi:10.1111/j.14710528.2001.00230.x

10. Ghalandari S, Kariman N, Sheikhan Z, Shahrahmani H, Asadi N. Systematic review on variety of effective treatment methods for postpartum hemorrhage in Iran and world. Iran J Obstet Gynecol Infertil. 2016;19(15):16-38. [Persian].

11. Hofmeyr GJ, Abdel-Aleem $\mathrm{H}$, Abdel-Aleem MA. Uterine massage for preventing postpartum haemorrhage. Cochrane Database Syst Rev. 2013(7):CD006431. doi:10.1002/14651858.CD006431.pub3

12. Kataoka Y, Nakayama K, Yaju Y, Eto H, Horiuchi S. Comparison of policies for the management of care for women and newborns during the third stage of labor among japanese hospitals, clinics, and midwifery birth centers. Int J Childbirth. 2015;5(4):200-209. doi:10.1891/2156-5287.5.4.200

13. Stafford I, Dildy GA, Clark SL, Belfort MA. Visually estimated and calculated blood loss in vaginal and cesarean delivery. Am J Obstet Gynecol. 2008;199(5):519.e511-517. doi:10.1016/j. ajog.2008.04.049

14. Nene BM, Hiremath PS, Kane S, Fayette JM, Shastri SS, Sankaranarayanan R. Effectiveness, safety, and acceptability of cryotherapy by midwives for cervical intraepithelial neoplasia in Maharashtra, India. Int J Gynaecol Obstet. 2008;103(3):232236. doi:10.1016/j.ijgo.2008.07.016

15. Masuzawa Y, Kataoka Y, Nakamura S, Yaju Y. Cooling the lower abdomen to reduce postpartum blood loss: a randomized controlled trial. PLoS One. 2017;12(10):e0186365. doi:10.1371/journal.pone.0186365 
16. Mitchell JL, Stecher J, Crowson J, Rich D. Uterine cooling during cesarean delivery to reduce blood loss and incidence of postpartum hemorrhage: a randomized controlled trial [31]. Obstet Gynecol. 2015;125 Suppl 1:9S-10S. doi:10.1097/01. aog.0000463548.24459.52

17. Masuzawa Y, Yaeko K. Uterine activity during the two hours after placental delivery among low-risk pregnancies: an observational study. J Matern Fetal Neonatal Med. 2017;30(20):2446-2451. doi:10.1080/14767058.2016.1253 057

18. Pourjavad M, Akhlaghi F. FIGO Launches initiative to decrease deaths during childbirth. Iran J Obstet Gynecol Infertil. 2002;5:99-101. [Persian].

19. Torkestani F, Radpour L, Hdipour I, et al. Safe Maternal Program: Integrated Maternal Health Care. 7th ed. Iran: Ministry of Health and Medical Education; 2018.

20. Schulz KF, Altman DG, Moher D. CONSORT 2010 Statement: updated guidelines for reporting parallel group randomised trials. BMC Med. 2010;8:18. doi:10.1186/1741-7015-8-18

21. Mooshkbid Haghighi M, Kamjoo A, Pedooim P, Kamali P. Intramuscular oxytocin should it be injected as routine to control postpartum hemorrhage? Iran Journal of Nursing. 2001;13(25):8-14. [Persian].

22. Dabbaghi Gale T, Elmizadeh K, Moradi S, Rashvand Melli E. Comparison of intravenous oxytocin and oral misoprostol in reduction of postpartum hemorrhage. J Adv Med Biomed Res. 2012;20(81):1-8. [Persian].

23. Mojahed S, Aflatunian A, Khadem N, Dehghani Firouzabadi R, Karimi Zarchi M. An investigation into effectiveness of date (Rutab) on postpartum hemorrhage. Journal of Shahid Sadoughi University of Medical Sciences. 2012;20(2):159166. [Persian]

24. Mahdavian M, Golmakani N, Manssori A, Hosseinzadeh $H$. An investigation of effectiveness of oral dill extracts on postpartum hemorrhage. Iran J Obstet Gynecol Infertil. 2001;4:19-26. [Persian].

(C) 2021 The Author(s); This is an open-access article distributed under the terms of the Creative Commons Attribution License (http:// creativecommons.org/licenses/by/4.0), which permits unrestricted use, distribution, and reproduction in any medium, provided the original work is properly cited. 